\title{
Feasibility study of injection mouldable conductive plastic for the hearing aid applications
}

\author{
Merca, Timea D.den; Lindberg, Torbjörn; Islam, Aminul
}

Published in:

Proceedings of the 11th International Conference on Multi-Material Micro Manufacture (4M2016) : co-organised with 10th International Workshop on Microfactories (IWMF2016)

Publication date:

2016

Document Version

Peer reviewed version

Link back to DTU Orbit

Citation (APA):

Merca, T. D. D., Lindberg, T., \& Islam, A. (2016). Feasibility study of injection mouldable conductive plastic for the hearing aid applications. In Proceedings of the 11th International Conference on Multi-Material Micro Manufacture (4M2016) : co-organised with 10th International Workshop on Microfactories (IWMF2016) (pp. 4). Research Publishing Services.

\section{General rights}

Copyright and moral rights for the publications made accessible in the public portal are retained by the authors and/or other copyright owners and it is a condition of accessing publications that users recognise and abide by the legal requirements associated with these rights.

- Users may download and print one copy of any publication from the public portal for the purpose of private study or research.

- You may not further distribute the material or use it for any profit-making activity or commercial gain

- You may freely distribute the URL identifying the publication in the public portal 


\title{
Feasibility study of injection mouldable conductive plastic for the hearing aid applications
}

\author{
Timea D.Merca ${ }^{1}$ Torbjörn Lindberg ${ }^{2}$ Aminul Islam ${ }^{3}$ \\ ${ }^{1}$ Department of Mechanical Engineering, Technical University of Denmark, Produktionstorvet, Building 427A \\ DK-2800 Kgs. Lyngby, Denmark \\ ${ }^{2}$ GN Store Nord Lautrupbjerg 7, 2750 Ballerup, Denmark
}

\begin{abstract}
Electrically conductive polymers can combine the advantage of plastic processing with the unique electrical properties which are usually found in metals. This article presents a feasibility study of an electrically conductive plastic for hearing aid antennas. Focus will be placed to critically analyse the electrical properties of the potential conductive plastic in a two component injection moulding process chain. The purpose of this experimental study is to mimic the real scenario in a hearing aid device and conclude the antenna's efficiency based on the results obtained with OTA (over the air) 3D measuring system in comparison with an ideal copper antenna at $2.4 \mathrm{GHz}$. An analysis of the association between the conductive plastic processing parameters in regards to its electrical performance is discussed and evaluated.
\end{abstract}

Keywords: Hearing Aids, Electrically Conductive Polymers, Comparative analysis.

\section{Introduction}

Conductivity is a property that mainly corresponds to metals; therefore, in order to achieve conductivity in polymers, these materials should behave as metals, meaning that its electrons need to move freely instead of sticking to the atoms.

A way to obtain electrical properties in polymers is by filling a non-conductive matrix with an electrically conductive material. The conductive fillers can be metals, carbon black particles, carbon nanotubes, etc. This paper focusses on a metal filled polymer due to its high electrical conductivity that could lead to a promising efficiency in a $2.4 \mathrm{GHz}$ antenna.

Another possibility is the use of intrinsically conductive polyaniline polymers, PEDOT (Poly 3, 4ethylenedioxthiophene Poly Styrene Sulfonate), PPy, etc. or conductive carbon nanotube (CNT); alternatives usually available in thin films, which have been studied in antenna applications with successful results even with lower conductivity levels. In this precise case, a thermoplastic has been chosen using CES Material Selector software, particularly the most conductive commercially available material that encloses the initial requirements of a two component moulding application.

Currently, a copper antenna ensures a sufficient electrical conductivity. However, the effectiveness of a conductive polymer with a significant lower electrical conductivity, can be debated. Additionally, one of the biggest challenges of small antennas in hearing aids with limited space allowance is the increment of magnetic fields. This is due to the proximity between the antenna traces, which influences the electrical losses, thus, decreasing the efficiency of the device.
Another way to combine metal and polymer successfully, is seen in the process chains for MID's (moulded interconnect devices). One of these processes is moulding two different polymers by two component injection moulding, in which one of the $2 \mathrm{~K}$ parts, receives a posterior electroless metal deposition (currently already applied in mobile phone antenna and other electrical circuits). Another process consists of an electrical metal deposition of a plateable surface activated previously by a laser [1].

The aim of this paper is to give a step forward into the simplification of the aforementioned process chains for MID's, eliminating the metallization process as well as the use of the laser. This can be done by combining a conductive trace in a non-conductive matrix during initial moulding. However, the main analysis of this paper is focused on the antenna application performance and not the process chain simplification.

\section{Material and method}

\subsection{Material}

The electrical conductivity level was the main criteria for the selection of the material in this study. The chosen polymer was SCHULATEC® TinCo 50, produced by Schulman Europe, with a conductivity of $5 \cdot 10^{5} \mathrm{~S} / \mathrm{m}$ (whereas copper conductivity $59.1 \cdot 10^{6} \mathrm{~S} / \mathrm{m}$ ). This plastic-metal hybrid consist of SCHULAMID® 6 (PA) matrix material, copper fibre and a low-melting solder (Tin), in a percentage of $50 \%$ filler and $50 \%$ PA.

\subsection{Antenna demonstrator}

For the creation of the antenna prototypes, several plates of various thicknesses were injection moulded, 
with a later machining process for the creation of the antenna patterns.

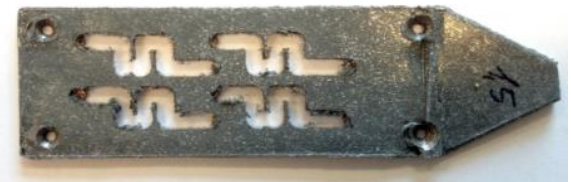

Fig. 1. TinCo 50 plastic plate with the antenna patterns cut along the $X$ axis. Length of each antenna: $30 \mathrm{~mm}(\lambda / 4)$.

Table 1 shows the processing parameters used for the fabrication of the four different plates with different thicknesses: $0.5,1,1.5$ and $2 \mathrm{~mm}$.

Table 1

Processing parameters of the moulding of the plates

\begin{tabular}{lcccccc}
\hline Parameter & Units & & & & \\
\hline Plate thickness & $\mathrm{mm}$ & 0.5 & 1 & 1.5 & 2 \\
\hline $\begin{array}{l}\text { Melt temperature } \\
\text { in nozzle }\end{array}$ & $\stackrel{\circ}{ } \mathrm{C}$ & 235 & 235 & 235 & 235 \\
$\begin{array}{l}\text { Injection pressure } \\
\text { Injection speed }\end{array}$ & $\mathrm{mm} / \mathrm{s}$ & 500 & 300 & 80 & 40
\end{tabular}

The processing parameters for the plates formation are mentioned due to the further relation with the conclusion of this study case. During the injection process, the major difference compared with the information provided by the supplier was the material melting temperature. A significant reduction of the processing temperature was needed since the material was leaking out of the cylinder in the feeding point: from $260-290^{\circ} \mathrm{C}$ to $235^{\circ} \mathrm{C}$ as listed in Table 1 .

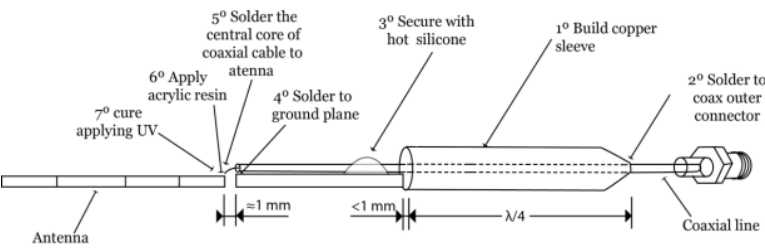

Fig. 2. Assembly sequence for antenna demonstrator.

As seen in Fig. 2. in a lateral view of the antenna demonstrator, from the right to the left, the prototype was composed of: the coaxial cable connected to the antenna by the internal core. The external part of the cable connected to the ground plane with the purpose of a return path of the current from the antenna. A copper choke aimed at balancing the signal variations caused by the coaxial cable due to a high proximity to the antenna. A ground plane cut from the same SCHULATEC TinCo 50 plate as the one used for each corresponding antenna. Finally, the antenna cut from the plates listed in Table 1, with a constant width of 1 $\mathrm{mm}$.

The soldering of the antenna to the internal core of the coaxial cable was made using a $\mathrm{Sn}-\mathrm{Ag}-\mathrm{Pb}$ solder agent. Additionally, the antennas were placed in ABS "shells" in order to mimic the electrical losses that the real scenario would introduce (Fig. 3).

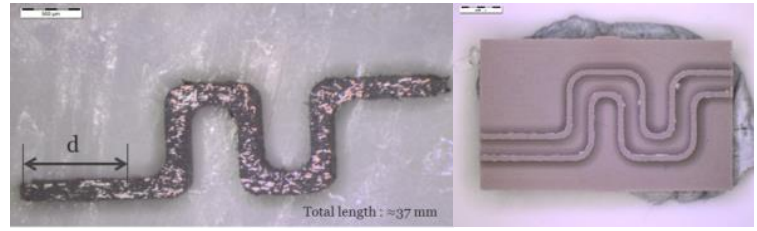

Fig. 3. Left: Antenna (d: section mechanically removed for $2.4 \mathrm{GHz}$ calibration). Right: ABS shell.

\section{Results and discussion}

\subsection{Resonance frequency analysis}

The first results of the return loss measurements during and after the antennas calibration, using an Agilent ENA-L RF Network Analyser, showed already a clear difference between the different cross section categories.
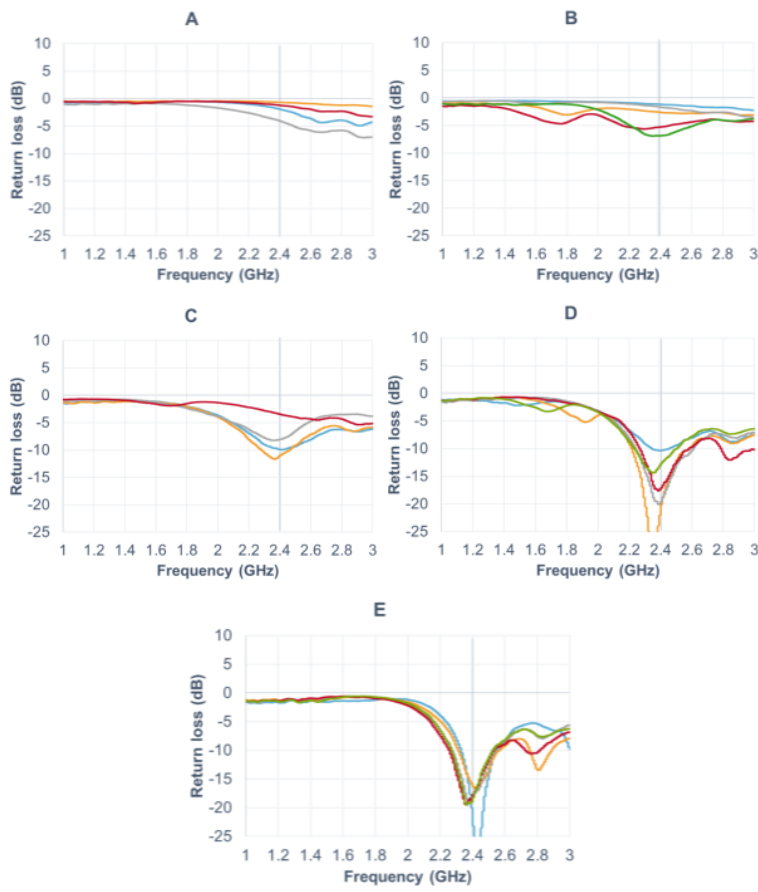

Fig. 4. Return loss of processed antenna. (A) $0.5 \mathrm{~mm}$ plate. (B) $1 \mathrm{~mm}$ plate. (C) $1.5 \mathrm{~mm}$ plate. (D) $2 \mathrm{~mm}$ plate. (E) Copper reference. (Measurements taken after frequency adjustment of $2.4 \mathrm{GHz}$ ).

As shown in the graphs in Fig. 4, there is a direct correlation between the thickness of the plates and the antennas performance. The return loss measurements of the antennas cut from the $2 \mathrm{~mm}$ plates have similar results to the reference copper antennas presented in Fig. 4 (E).

\subsection{OTA testing (Over-The-Air)}

To ensure a more accurate evaluation, aside from the return loss measurement, a $3 \mathrm{D}$ measuring test called OTA (over the air) was conducted in an anechoic chamber. This test involves a 3D spherical examination that measures the radiated power, gain, sensitivity, efficiency and many other parameters during the wireless performance of the device. These measurements consist of minimum and maximum records in a spherical far-field by analysing the antenna 
pattern every certain degree, using alternative polarisation methodology. Moreover, a dipole was also tested, using the same installation and connection cable.

The efficiency results shown in Fig.5, match the first return loss measurements, in which the $2 \mathrm{~mm}$ thickness antenna series presented the best performance. In the best result, the efficiency of the conductive polymer is only $10 \%$ lower than the copper reference. However, the results are not consistent within each thickness series. One of the reasons is that the antenna patterns, even if being cut from the same plate, have different metal distribution along the plate, which might have caused a different electrical behaviour. The areas closest or furthest away from the injection gate, have different metal concentrations, in which the metal proportion is increased in the areas further away from the gate.

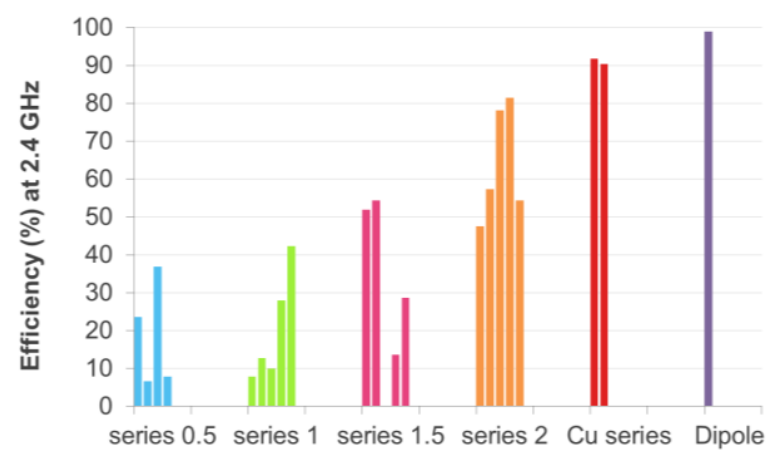

Fig. 5. Efficiency comparison between all series $(0.5 ; 1 ; 1.5$; 2; Cu; Dipole) at $2.4 \mathrm{GHz}$.

In the analysis above, the antennas efficiency values are based on the highest efficiency in a specific direction. The 3D radiation pattern (Fig. 8) shows how the metal distribution inside the polymer matrix influences the radiation of the antenna in all directions. Although all samples' radiation patterns were analysed during this study case, Fig. 6 and Fig. 7 show only some of the most representatives for understanding the results. The radiation patterns of the conductive plastic antennas show good uniformity and similar 3D space radiation as the copper reference as seen in Fig. 6.

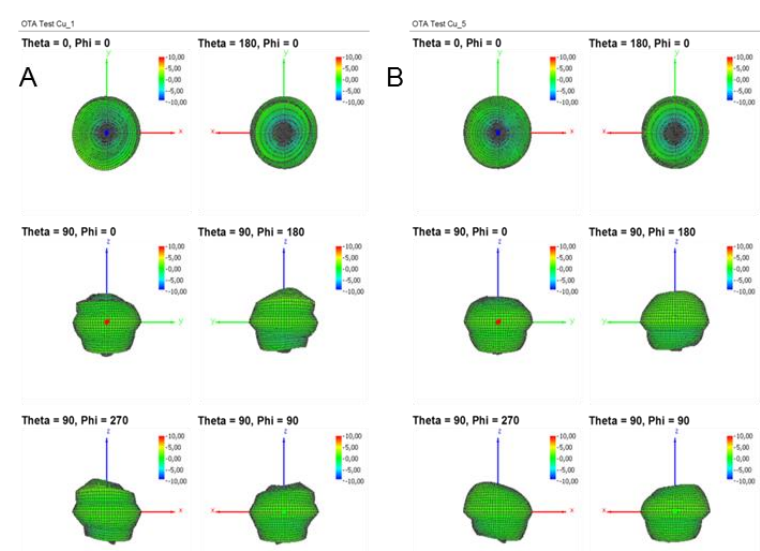

Fig. 6. 3D radiation patterns $(A)$ and $(B)$ copper reference.

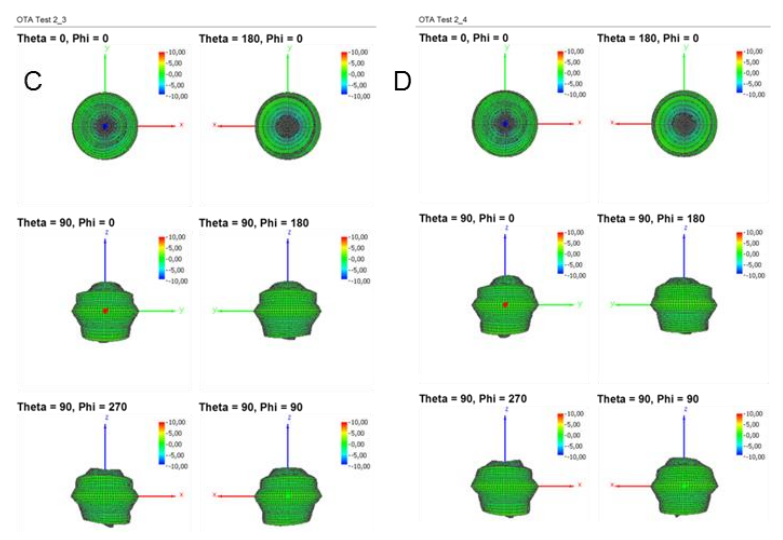

Fig. 7. 3D radiation patterns. (C) and (D) $2 \mathrm{~mm}$ series antenna.

The non-spherical shape of the radiations of the copper and the conductive plastic was considered to be caused by the physical shape of the antenna, in which no design criteria was followed for an optimum 3D radiation.

\subsection{Cross section investigation}

The reasons for which the antennas cut from the thicker plates had shown better results, can be derived through various factors: high-orientated metal distribution, aspect ratio and skin depth effect.

\subsubsection{High oriented metal distribution}

During processing, in order to achieve a complete filling of the parts, the injection speed was significantly increased in the thinner plates, as seen in Table 1. This parameter has influenced the metal distribution inside the samples. It is already known from previous studies, that the injection speed can reduce the electrical effectiveness because it leads to a highly-oriented and less uniform distribution of the metal fibres [2]. During soldering, the electrical connection was easily established in the antennas cut from the $1.5 \mathrm{~mm}$ and 2 $\mathrm{mm}$ plates unlike the 0.5 and $1 \mathrm{~mm}$ ones. This was due to the decrease of electrical conductivity of the thinner plates as measured by the multi-metre, proving the theory aforementioned.
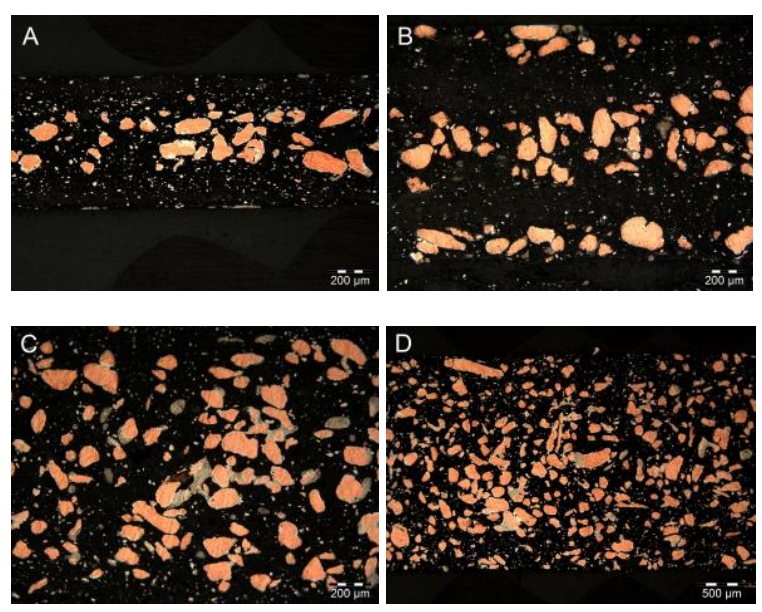

Fig. 8. Cross section of the filling direction. Area close to sample gate. (A) $0.5 \mathrm{~mm}$ plate. (B) $1 \mathrm{~mm}$ plate. (C) $1.5 \mathrm{~mm}$ plate. (D) $2 \mathrm{~mm}$ plate 
As can be seen in Fig. 8, the metal distribution within the four different plates is clearly uneven. While in picture $(C)$ and $(D)$, the metal fibres show a uniform distribution, in (A) and (B) both distribution and metal concentration is affected by the stresses during the injection moulding process. Moreover, the electrical connectivity seems to be also affected by the Tin and Copper dispersion. As stated by the material supplier, the Tin acts as a binder to the copper fibres in order to achieve electrical conductivity in the material. As seen in the figure below, the assumed Tin function was only achieved at lower injection speeds for the thicker plates.

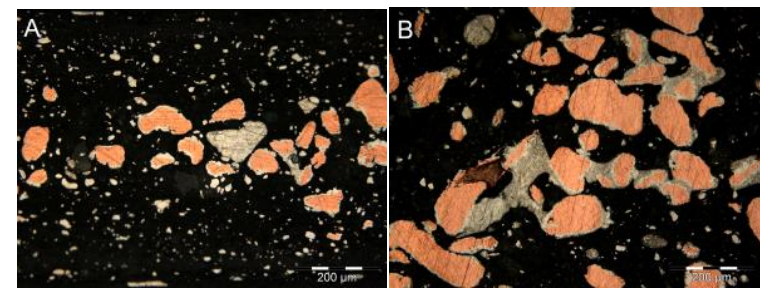

Fig. 9. Tin and Copper distribution. (A) $0.5 \mathrm{~mm}$ plate. (B) 1.5 $\mathrm{mm}$ plate. Black: PA matrix; Reddish brown: Copper; Light Grey: Tin

In the distribution examples shown in the Fig. 9, at higher injection speeds $(A)$, Tin is dispersed among the sample in both small and big particles and, overall, does not fulfil its binding function. It is observed to remain concentrated close to the sample's surface in form of small particles and possibly long fibres highly orientated to the injection flow as a result of the high injection speed. A good example of the Tin distribution for a good electrical connection is shown in picture (B), in which Tin has created a path between the copper fibres to allow the current flow.

\subsubsection{Aspect ratio}

The electrical conductivity is also related to shape of the filler particles, specifically to the aspect ratio (length-to-width ratio): for a high aspect ratio, the electrical conductivity increases [3]. In case of other metal fillers, studies have shown that the distribution and the aspect ratio of the fibres within the injected part, was different because of dissimilar flowing shear stress. The aspect ratio of the fibres at the skin layer is smaller than other layers because the flowing shear stress is the highest in this area [3]. In the core layer, usually the shear stress is lower, however, in this case, if the stresses were too high at high injection speeds, same effect might have occurred. Consequently, increasing the electrical resistivity and leading to a poor antenna performance. Further research on the shape of the fibres should be performed since this study only analysed the cross section areas in the plastic flow direction.

\subsubsection{Skin depth effect}

The skin depth is a phenomenon that occurs when AC current is applied to a conductor. In high frequencies, the electrical flow concentrates at the metal (usually wire) surface increasing in this way the electrical resistance. This is due to the amount of material inside the part through which the current is not flowing [4].
For AC applications, it is important to maintain the thickness in the range of the skin depth in order to avoid a resistance increment. In the case of Copper, the skin depth is around $1.3 \mu \mathrm{m}$ at $2.4 \mathrm{GHz}$, and 3.4 $\mu \mathrm{m}$ for Tin at the same frequency. After analysing the cross section areas (Fig. 8), the size of some metal fibres within the matrix was found to be higher than these values, sometimes even close to $200 \mu \mathrm{m}$. Consequently, the skin effect in these areas might have originated losses due to the high concentration of currents on the outer areas of the metal.

\section{Conclusions}

The electrical conductivity of Schulatec TinCo 50 has proven to be an important factor in antenna application. The antenna performance showed a considerably lower efficiency for a higher electrical resistivity. Further electrical test are required to quantify the electrical losses.

The lower the injection speed, the better the antenna performance, as a result of a more uniform metal distribution and higher electrical conductivity. By reducing the injection speed, and with it the internal stresses, the Tin showed an optimum binding to the less orientated copper fibres. This creates a network through which the current is allowed to flow more optimally among the material.

The radiation patterns of the Schulatec TinCo 50 antennas had shown good uniformity in 3D space, as well as sufficient gain $(\mathrm{dB})$ values in the threedimensional space with similar results to copper. The difference was approximately $10 \%$, however, with further optimizations, this value could possibly be optimized.

\section{Acknowledgements}

This paper report was part of a practical investigation carried out in collaboration between the Department of Manufacturing Engineering (DTU) and GN Resound A/S, international developer and manufacturer of hearing healthcare solutions.

Both institutions are acknowledged for their help, support and provision of all materials and equipment required for the accomplishment of this experimental study.

\section{References}

[1] Aminul, Islam, "Two component micro injection moulding for moulded interconnect devices," Kongens Lyngby, 2008.

[2] X. Sun, Y. Cheng and H. Wu, "Research on Microstructure and Electrical Resistivity of Injection Molded Metallic Fiber-Filled Polymer Composites", AMR, vol. 472-475, pp. 748-752, 2012.

[3] Vlasveld D, Hurst D, Martínez P. "'Processing of conductive polymer composites. An introduction to conductive polymer composites, iPolyCond, ed. Smithers Rapra Technology, pp. 23-32, 2011.

[4] Akhilesh Verma, Christophe Fumeaux, "Effect of film thick-ness on the radiation efficiency of a 4.5 $\mathrm{GHz}$ polypyrrole con-ducting polymer patch antenna," Asia-Pacific Microwave Conference Proceedings, pp. 95-98, 2010. 